\title{
Dehiscence of a bioprosthetic mitral valve without endocarditis: A case report
}

\author{
Loren Francis* and Eric Nelson \\ Medical University of South Carolina, Charleston, USA
}

\begin{abstract}
Perivalvular leak is a serious, yet uncommon complication of prosthetic mitral valve replacement. 1-3\% of these patients require re-operation due to development of symptoms of heart failure and/or hemolysis. We present a patient who returned to the hospital in fulminant heart failure 4 months after an MVR due to a perivalvular leak not caused by endocarditis. We discuss the typical pathology and implications of a prosthetic valve dehiscence and the challenges these patients present in the perioperative period along with the clinical significance of perivalvular leak not due to endocarditis.
\end{abstract}

\section{Introduction}

Perivalvular leak due to valve dehiscence is a serious, yet uncommon complication of mitral valve replacement surgery. It is estimated to occur in $7-17 \%$ of patients, with $1-3 \%$ of patients requiring reoperation due to symptoms of heart failure [1,2]. The vast majority of patients who develop a valve dehiscence have endocarditis. Typically only about $3 \%$ of patients with valvular dehiscence do not have an aspect of endocarditis.

Patients with a severe enough perivalvular leak who present in heart failure require preoperative optimization including diuresis, and at times mechanical support to decrease morbidity and mortality and improve postoperative outcomes.

Transesophageal echocardiography (TEE) is useful in the perioperative period in order to identify and define where the leak is and to assess the repair postoperatively. TEE can also be used to assess volume and LV function to help guide management intra and postoperatively. Written consent has been obtained from the patient.

\section{Case}

A 50-year-old man presented 4 months after mitral valve replacement which was originally performed because of severe ischemic mitral regurgitation with ruptured chordae, with symptoms of heart failure. A transthoracic echocardiogram revealed a bioprosthetic mitral valve "rocking" in position with anteromedial instability and severe perivalvular regurgitation.

The patient was admitted to the hospital and quickly decompensated into cardiogenic shock with complications including atrial flutter, kidney impairment, liver injury, and evidence of hemolysis. An Impella left ventricular assist device was placed to augment cardiac output. His clinical condition improved enough that the Impella was removed and replaced with an intraaortic balloon pump (IABP). The patient was deemed a surgical candidate for mitral valve replacement and he was brought to the operating room.

Intraoperative TEE revealed a bioprosthetic trileaflet valve in the mitral position with anteromedial instability and a severe perivalvular leak. There was no evidence of endocarditis to the valve or the annulus. TEE also revealed a moderately enlarged right ventricle with moderate dysfunction and decreased left ventricular ejection fraction (LVEF).

The surgeon repaired the perivalvular leak with pledgeted sutures and upon determination of no further valvular instability the patient was weaned off CPB with the aid of an IABP, epinephrine, vasopressin, milrinone, and norepinephrine infusions. The patient was transferred to the intensive care unit, continued to improve and was discharged to home on postoperative day 12 (Figures 1-5).

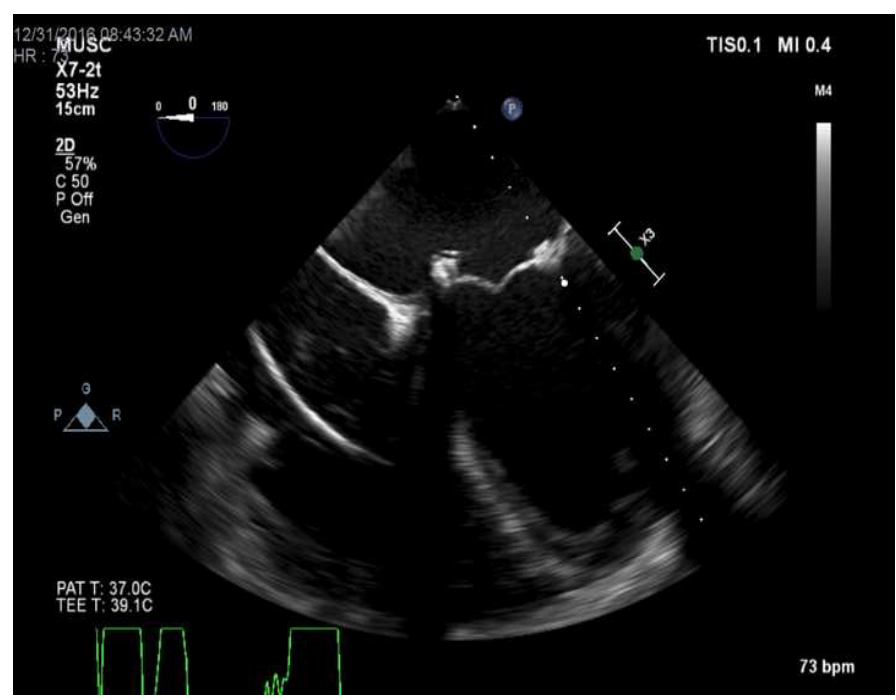

Figure 1. TEE - ME 4 chamber view demonstrating medial dehiscence of bioprosthetic mitral valve.

Correspondence to: Loren Francis, M.D. Medical University of South Carolina, 25 Courtenay Drive, Room 4200, Charleston, SC 29425, USA, Tel: (843) 876 5744; E-mail: francil@musc.edu

Received: October 03, 2017; Accepted: November 14, 2017; Published: November 17,2017 


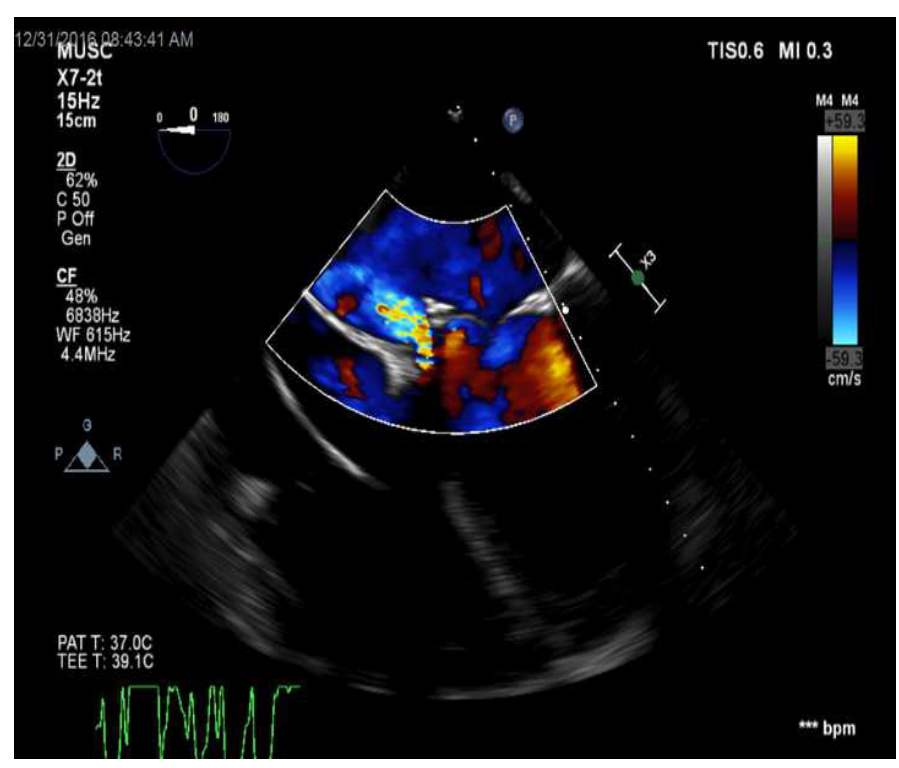

Figure 2. TEE - ME 4 chamber view demonstrating regurgitant flow through perivalvular leak.

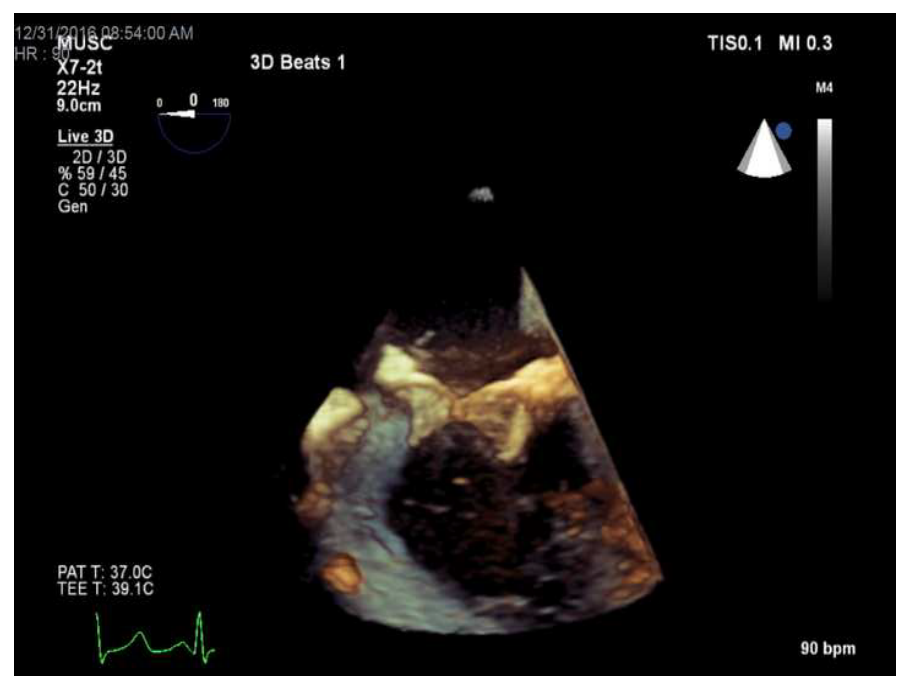

Figure 3. 3D TEE view of bioprosthetic mitral valve with dehiscence.

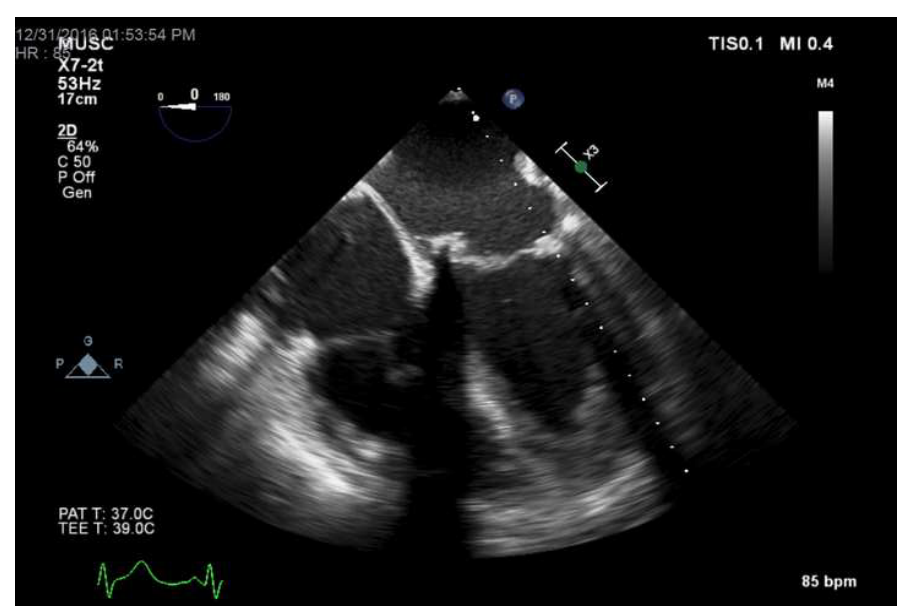

Figure 4. TEE - ME 4 chamber view of closure of perivalvular leak.

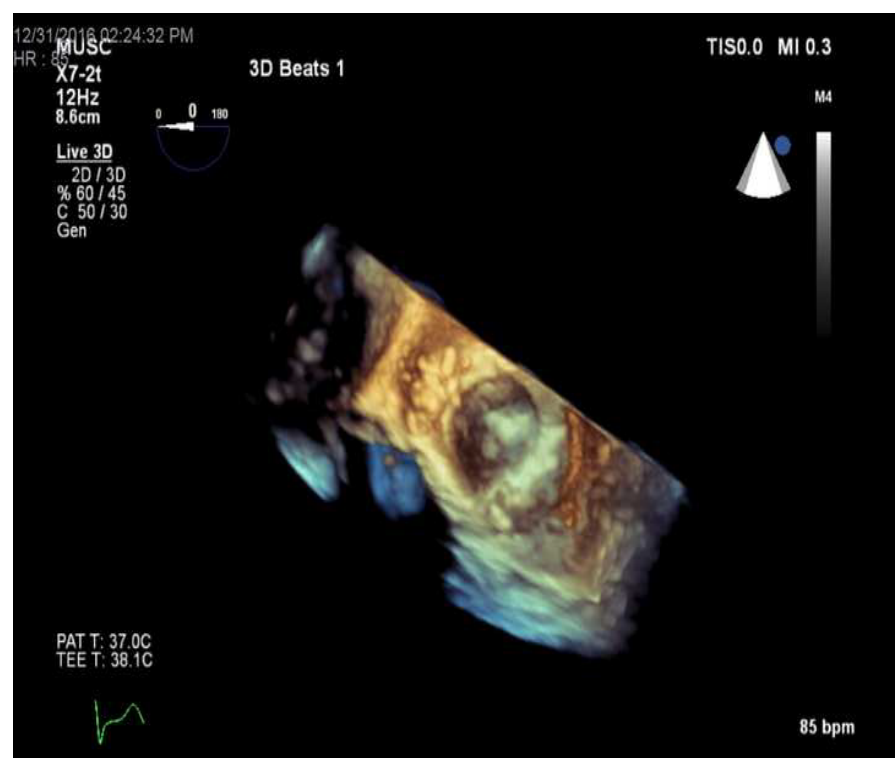

Figure 5. 3D TEE en face view of plegeted sutures closing perivalvular leak.

\section{Discussion}

Perivalvular leak is a serious yet uncommon complication of prosthetic mitral valve replacement estimated to occur in $7-17 \%$ of patients. Only 1-3\% of those require reoperation for development of heart failure symptoms and/or hemolysis [1].

Endocarditis is by far the most common cause of prosthetic mitral valve dehiscence, as infected and inflamed tissue surrounding the valve becomes increasingly friable and easily torn. One retroactive study of 435 patients after mitral valve replacement showed a $3.4 \%$ rate of developing perivalvular leak without endocarditis [3]. Another study showed a $2.5 \%$ incidence of perivalvular leak over 15 years in one hospital, and $79 \%$ of those involved infection [4]. Calcification of the mitral annulus and duration of cardiopulmonary bypass are potential risk factors for developing perivalvular leak [5].

Risk factors unrelated to infection may include choice of suture and technique during the original mitral valve replacement. Smaller thickness sutures may have a cutting effect on tissues or break over time. Semicontinuous suturing technique creates a higher risk of developing perivalvular leak as compared to interrupted sutures [6]. Interestingly, it is more common for mitral valve dehiscence to be posterior, possibly because it is the furthest from surgeon view with more prevalent calcifications and closest to the circumflex artery which surgeons attempt to avoid [7].

A patient who develops a perivalvular leak with a prosthetic mitral valve may suffer from heart failure secondary to acute mitral regurgitation [8]. The severity of the patient's condition depends on the acuity of regurgitation, as the heart does not have time to adapt to the hemodynamic changes. The left atrium sees an increase in volume and pressure, which leads to pulmonary edema. Forward flow decreases, causing cardiogenic shock. Patients who suffer this complication often present with a combination of congestive heart failure and/or hemolysis, both of which are indications the patient may need a second procedure [1]. 
Preoperatively patients may require medical optimization. One goal of care is to reduce afterload in order to augment forward flow, to increase end organ perfusion, and to decrease pulmonary edema. Inotropes or at times an IABP or temporary left ventricular assist device may be necessary to augment LVEF.

Intraoperatively, a TEE exam is often helpful in surgical planning. Ruling out endocarditis is important, as an infection would lead to replacement of the valve versus being able to perform a repair if there is merely a dehiscence. TEE is also useful to aid in coming off bypass for volume management and inotropic support. Additionally, an echo can help guide placement of assist devices such as intra aortic balloon pumps and Impella left ventricular assist devices.

\section{Conclusion}

This patient suffered from an uncommon complication of mitral valve replacement, and it caused him to become extremely ill prior to surgical repair. It was important to medically optimize him prior to surgery. The anesthesia, surgical, and cardiology teams worked together to plan for this procedure. In particular, the real time TEE exam helped confirm the diagnosis and guide planning for correction.

\section{References}

1. Ruiz CE, Jelnin V, Kronzon I, Dudiy Y, Del Valle-Fernandez R, et al. (2011) Clinical outcomes in patients undergoing percutaneous closure of periprosthetic paravalvular leaks. J Am Coll Cardiol 58: 2210-2217. [Crossref]

2. Rihal CS, Sorajja P, Booker JD, Hagler DJ, Cabalka AK, et al. (2012) Principles of percutaneous paravalvular leak closure. JACC Cardiovasc Interv 5: 121-130. [Crossref]

3. Dhasmana JP, Blackstone EH, Kirklin JW, Kouchoukos NT (1983) Factors associated with periprosthetic leakage following primary mitral valve replacement: with special consideration of the suture technique. Ann Thorac Surg 35: 170-178. [Crossref]

4. Jindani A, Neville EM, Venn G, Williams BT (1991) Paraprosthetic leak: a complication of cardiac valve replacement. J Cardiovasc Surg (Torino) 32: 503-508. [Crossref]

5. Wąsowicz M, Meineri M, Djaiani G, Mitsakakis N, Hegazi N et al. (2011) Early complications and immediate postoperative outcomes of paravalvular leaks after valve replacement surgery. J Cardiothorac Vasc Anesth 25: 610-614. [Crossref]

6. Nair SK, Bhatnagar G, Valencia O, Chandrasekaran V (2010) Effect of valve suture technique on incidence of paraprosthetic regurgitation and 10-year survival. Ann Thorac Surg. 89: 1171-1179. [Crossref]

7. Kronzon I, Sugeng L, Perk G, Hirsh D, Weinert L, et al. (2009) Real-time 3-dimensional transesophageal echocardiography in the evaluation of post-operative mitral annuloplasty ring and prosthetic valve dehiscence. J Am Coll Cardiol 53: 15431547. [Crossref]

8. Gaasch WH, Meyer TE (2008) Left ventricular response to mitral regurgitation: implications for management. Circulation 118: 2298-2303. [Crossref]

Copyright: (C2017 Francis L. This is an open-access article distributed under the terms of the Creative Commons Attribution License, which permits unrestricted use, distribution, and reproduction in any medium, provided the original author and source are credited. 